\title{
Modification and Actuator Minimization of the Hip Leg Joint in a Bipedal Robot, a Proposed Design
}

\author{
Nirmalya Tripathi*, Ajay Tiwari** \\ * Department of Mechanical Engineering, University of Petroleum and Energy Studies, India \\ ** Department of Mechanical Engineering, Rungta College of Engineering and Technology, India
}

\begin{abstract}
Article Info
Article history:

Received Feb 9, 2015

Revised Apr 25, 2015

Accepted May 12, 2015

\section{Keyword:}

Actuators

Herringbone gear

Microcontroller

Minimize

VR DC Stepper Motor

ABSTRACT

In recent times, there have been numeric applications of Biped Robots. In this paper, a proposed upper leg hip design of a biped was developed taking cost reduction and optimization as factors for consideration. The proposed system introduces a novel method which consists of a vibration reduction (VR) DC stepper motor, microcontroller, microprocessor and gearing arrangement. The program in the microprocessor is so designed that it gives a fixed number of cycles/steps to the VR DC stepper motor in clockwise and thereafter in anti-clockwise direction. This turning movement can then be transmitted to the gearing system which precisely moves one upper leg when the VR DC stepper motor moves in clockwise direction, while the other upper leg remains static, and vice-versa. It has been observed that this new proposed system may reduce the cost overhead, weight and the energy consumption incurred by working on a single VR DC stepper motor while conventionally two stepper motors are used to give the motion of the two upper legs in a biped.
\end{abstract}

Copyright $(0) 2015$ Institute of Advanced Engineering and Science. All rights reserved.

\section{Corresponding Author:}

Nirmalya Tripathi, Department of Mechanical Engineering, University of Petroleum and Energy Studies, Energy Acres, P.O. Bidholi, Dehradun, 248007, India.

Email: ntripathi@ddn.upes.ac.in

\section{INTRODUCTION}

A biped robot is a robot that simulates the activities of a human being. The bipeds have numerous applications in various fields such as inspection, defense, manufacturing, cleaning, remote exploration, and entertainment [Hudyjaya Siswoyo Joa * et al 2012]. A biped robot has similar movements to that of a human being [Ronald Van Ham* et al 2007 ]. It should have a gait pattern similar to that of a human gait, hence it needs actuators for its movement [Koh Hosod ${ }^{*}$ et al 2008]. In a biped robot, the motion of the actuators in each joint is achieved by using separate DC stepper motors. Numerous motors are used to provide the motion to every joint. The movement of a conventional biped robot leg is periodical. The upper leg links of the hip joint have two separate DC motors for the individual link movement. It ensures the precise and definite movement to each of the leg module. Formerly various novel work has been done in this area to reduce the overall weight, energy consumption and optimize the overall system in a biped [Ronald Van Ham* et al 2007]. Sung-nam Oh et al had designed the biped which is monitored and controlled by a computer and the mechanical system consisted of links and joints with more than 2 DOF with a single DC motor giving only one direction of motion. Hudyjaya Siswoyo Jo et al had also developed a novel way to minimize the number of actuations and had used two motors in the hip joint for the motion of the legs. R. Van Ham et al had proposed the actuation of the hip and the leg joint with a simple spur gear arrangement and focused on the precise and control movement of the biped. K. Hosoda et al proposed and experimented a novel design with 
two pneumatic actuators for the locomotion of the biped. M. Guihard, P. Gorce et al had had also proposed a biped robot using pneumatic actuators and emphasized on the dynamic behavior of the leg.

This paper proposes a new design and control approach which is built upon mechanisms and hardware involved in the hip joint module considering less weight, minimal hardware, less cost and reduce energy consumption as factors. The program in the control system provides the stepper motor actuation of the hip joint in both clockwise and anti-clockwise. The clock wise motion is transmitted to one leg while the other leg remains stationary and vice versa. This motion is achieved by a new gear arrangement design. The results of the mechanical design was verified in virtual simulation and the simple control system was experimentally validated.

\section{RESEARCH METHOD}

The hip leg design is an important area in biped robots as it involves the static and dynamic stability, and the motion transmission. Many novel works has been done in the design of the hip and leg in bipeds. The belt train drives are mostly used for the motion transmission from the DC stepper motors to the leg [M. Reza Emami et al 2014].

The proposed approach is designed to reduce the mechanical structure of the upper leg. In the design, the movement of both the upper leg of a biped robot has been tried to achieve with a single DC stepper motor. This is done by rotating the DC stepper motor discretely in both directions (clockwise and anti-clockwise). The DC stepper motor is connected by the microcontroller and a programmed microprocessor. This separate directional motion is utilized with necessary gear arrangements to move both the upper leg links of the hip joint one at a time. The gear arrangement is designed using the software Catiav5 developed by Dessault systems. This paper is settled with the design and control of planar bipeds which can walk in only in one direction, either forward or backward with a single DC motor.

\subsection{Control System}

The control system was developed keeping cost and easy operation in mind while the conventional functionality is not impacted in the biped robot. The entire system developed is represented by the block diagram (Figure 1). The control system consisted of a microcontroller, microprocessor, and vibration reduction DC stepper motor. The operational codes were first programmed in assembly language as per the requirement. It was then fed manually to the microcontroller. The microcontroller was connected to the microprocessor which processed the set of codes in the microprocessor and thereby sent electric pulse signals to the DC stepper motor connected, which then rotates for the defined stops as provided in the operational codes. The idling time was specified in the code before the stepper motor rotates in the opposite direction. The specific rotation could be changed in accordance to the requirement in the real time process. This motion of the DC stepper motor can then be transmitted to the new gear train designed which would give motion to both the upper leg links of the hip joint of the biped robot. There were two set of operational codes that was programmed. The first set of codes gives the clock wise motion to the stepper motor while the second set of codes was giving the anti-clock wise motion to the same DC stepper motor. This would reduce the use of the second stepper motor that provided the motion to the other leg. The operational code that was programmed is mentioned below and their functionality is then discussed. The configuration of the control system is specified below.

1. Stepper motor - TYPE - STM-601, INPUT/VOLTAGE - 12V, DC/AMP - 0.5A, TORQUE - $2 \mathrm{~kg}$

2. Micro controller - TYPE - 8255

3. Microprocessor - TYPE - DYNA-85

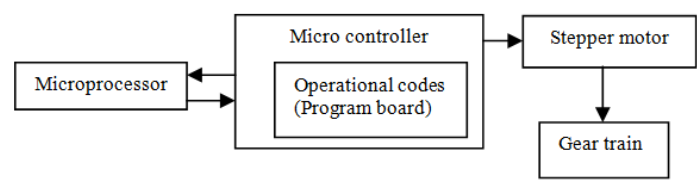

Figure 1. The entire developed system

\subsubsection{Operational Code Program}

Coding for the micro-processor to execute the motor for 8 steps (first in clock wise direction and then in anti-clockwise direction) 
C000 MVIA, $80 \mathrm{H}$ IIThis statement loads the 8255 control word in the Accumulator. All the ports are output ports.

C002 OUT 13H IIThis sends the control word address from the micro controller to the microprocessor.

C004 MVIC,08H IIThe resistor is used as a counter,8 is the number of count of steps in clockwise of the motor.

C006 MVIA,FA IVLoad FA command in the Accumulator to excite the magnetic effect in the stepper motor.

C008 OUT $10 \quad$ IIPort A is accessed to send the command.

C010 CALL 05,F1 IVelay the program to run the previous step.

C013 MVIA,F6 IILoad the F6 command.

C015 OUT $10 \quad$ IIPort A is accessed.

C017 CALL 05,F1 IIDelay the program to run the previous step.

C020 MVIA,F5 IILoad the F6 command.

C022 OUT $10 \quad$ IIPort A is accessed.

C024 CALL 05,F1 INDelay the program to run the previous step.

C027 MVIA,F9 IVLoad the F6 command.

C029 OUT 10

IIPort A is accessed.

C031 CALL 05,F1 IVDelay the program to run the previous step.

C034 DCRC

C035 JNZ C006

INDecrement Resistor Count.

IVump Not Zero until C006.

The above machine level program runs for the clock wise rotation/cycle or steps for the DC motor. The continuation of the below machine level program is designed for the anti-clockwise rotation/cycle or steps for the DC motor.

C037 MVIC,08H IIThe resistor is used as a counter, 8 is the number of count of steps in anti-clockwise of the motor.

C039 MVIA,F9

C041 OUT 10

IVLoad the F9 command.

IIPort $\mathrm{A}$ is accessed to send the command.

C043 CALL 05,F1

C046 MVIA,F5

C048 OUT 10

C050 CALL 05,F1

IIDelay the program to run the previous step.

IVLoad the F5 command.

IVPort A is accessed to send the command.

IIDelay the program to run the previous step.

IILoad the F6 command.

IVPort $\mathrm{A}$ is accessed to send the command.

C055 OUT 10

C057 CALL 05,F1

IIDelay the program to run the previous step.

IILoad the FA command.

IIPort A is accessed to send the command.

C062 OUT 10

C064 CALL 05,F1

IVDelay the program to run the previous step.

C067 DCRC

INDecrement Resistor Count.

C068 JNZ C006

IVump Not Zero until C006.

C071 HALT IIStops the program.

OPT CODE (for clock-wise steps/cycles)

3E 80 D3 13 0E 08 3E FA D3 10 CD F1 05 3E F6 D3 10 CD F1 05 3E F5 D3 10 CD F1 05 3E F9 D3 10 CD F1 $050 \mathrm{D}$ C2 $06 \mathrm{C} 0$

OPT CODE (for anti-clock wise steps/cycles):-

0E 08 3E F9 D3 10 CD F1 05 3E F5 D3 10 CD F1 05 3E F6 D3 10 CD F1 05 3E FA D3 10 CD F1 05 0D C2 $06 \mathrm{C} 0 \mathrm{CF}$

\subsection{Design System}

\subsubsection{The Gear Mechanism}

The gear arrangement transmits motion to the upper leg links of the hip joint in the biped robot. Many novel works has been done in the transmission of the motion from the motor using belt-chain drive. The gear arrangement was designed considering two factors- (1) the motion would be more precise and (2) the fulfillment of the idea of using one stepper motor to move both the legs. The gear arrangement consisted of a compound gear train [Ronald Van Ham* et al 2007] and a reverted gear train [Ronald Van Ham* et al 2007]. There are 3 compound gears that were designed using Catiav5, one driving gear and two driven gears. The first gear that would attach directly to the stepper motor (called as driving gear) was designed seeing a herringbone gear profile. The profile was chosen as this would give motion in only one direction. The herringbone gear profile was designed in this driving gear, both in the outer peripheral plane and also in the radial plane [Figure 2]. There after another similar herringbone gear was designed (called as driven gear). 
The outer peripheral plane of this first driven gear was designed in spur gear arrangement and the radial plane was designed in herringbone profile [Figure 3]. This radial herringbone gear profile of this first driven gear meshes with the radial herringbone profile of the driving gear. There after the second driven gear is designed where outer periphery had spur gear profile and the inner periphery had herringbone gear mesh [Figure 4]. This inner periphery of this second driven gear meshes with the herringbone peripheral gear arrangement of the driving gear.

In the radial plane of this driving gear, a similar herringbone driven gear was designed paired whose outer cylindrical plane has spur gear arrangement. This spur gear is attached to another pinion spur gear where the upper leg link of the hip joint is attached.

This mechanism would provide motion to one driven gear while the other remains stationary. Again when the driving gear rotates in the opposite direction the former driven gear would be stationary while the other driven gear rotates.

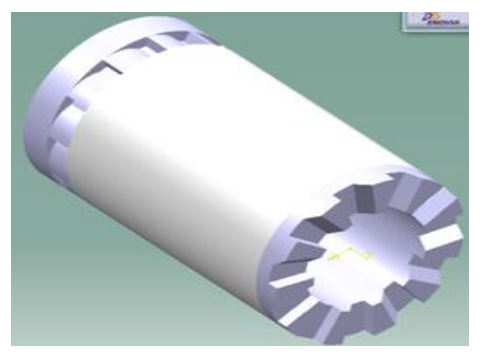

Figure 2. Driving gear

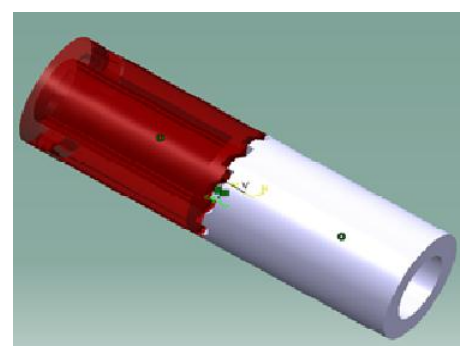

Figure 3. Radial plane

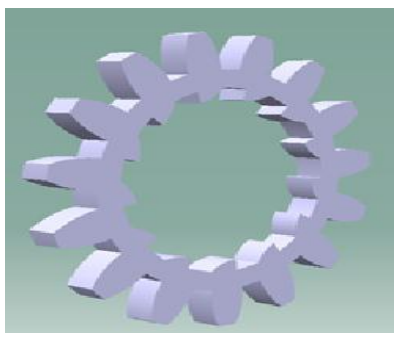

Figure 4. Herringbone gear mesh

The entire mechanism of the driving gear and the two driven gears is given in Figure 5.

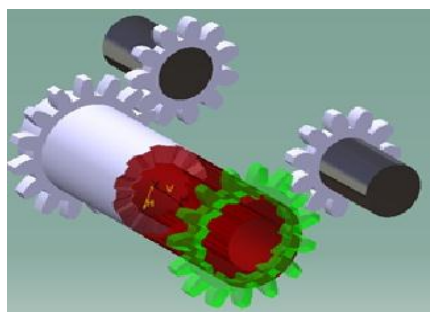

Figure 5. The entire mechanism of the driving gear and the two driven gears

\section{RESULTS AND ANALYSIS}

The control system has been verified by implementing the programmed operational codes in the microcontroller. It has been observed that the DC stepper motor was giving the specific rotation in both the directions (clock wise and anti-clock wise) as programmed. The number of steps was changed in the operational codes and the each experiment was repeated. The gear arrangement was designed successfully in Catia-v5 and the virtual simulation was also conducted. This was in accordance to the rotation by the stepper motor. 


\section{CONCLUSION}

In this paper, a new novel method has been proposed that might decrease the overall weight of the biped. The number of DC stepper motors can be minimized in various parts of the robot such as arm joint, leg joint, this would reduce the overall cost and the energy consumption of a biped. The new adaptive gear design can be incorporated in other parts to give precise movement. This paper restricts in manufacturing of this new gear train and can be further studied. The various material properties of the gear that can affect the performance can also be considered. The new gear design would lead to changes in the dynamic and static stability of the mechanical structure of the biped which can be further explored.

\section{REFERENCES}

[1] Victor Ragusila, M. Reza Emami, "Modelling of a robotic leg using bond graphs," Simulation Modelling Practice and Theory, vol. 40, pp. 132-143, 2014.

[2] Hudyjaya Siswoyo Joa, Nazim Mir-Nasiria, "Stability Control of Minimalist Bipedal Robot in Single Support Phase," International Symposium on Robotics and Intelligent Sensors, vol. 41, pp. 113-119, 2012.

[3] Erika Ottaviano , Sergey Vorotnikov, Marco Ceccarelli, Pavel Kurenev b "Design improvements and control of a hybrid walking robot," Robotics and Autonomous Systems, vol. 59, pp. 128-141, 2011.

[4] Koh Hosod, Takashi Takuma, Atsushi Nakamoto, Shinji Hayashi, "Biped robot design powered by antagonistic pneumatic actuators for multi-modal locomotion," Robotics and Autonomous Systems, vol. 56, pp. 46-53, 2008.

[5] Seungsuk Ha, Youngjoon Han, and Hernsoo Hahn, "Adaptive Gait Pattern Generation of Biped Robot based on Human's Gait Pattern Analysis," World Academy of Science, Engineering and Technology, vol.10, pp.10-27, 2007.

[6] Ronald Van Ham, Bram Vanderborght, Michael Van Damme, Bjorn Verrelst, Dirk Lefeber, "MACCEPA, the mechanically adjustable compliance and controllable equilibrium position actuator: Design and implementation in a biped robot," Robotics and Autonomous Systems, vol. 55, pp. 761-768, 2007.

[7] Sung-nam Oh, Sung-Ui Lee, and Kab-ll Kim, "Design of a Biped Robot Using DSP and FPGA," International Journal of Control, Automation, and Systems, vol. 1, pp. 252-256, 2003.

[8] M. Guiharda, P. Gorcea, "A new controller adapted to constrained pneumatic multichain structures," Automatica, vol. 36, pp. 1321-1327, 2000. 